\title{
Integration of Lean Approaches to Manage a Manual Assembly System
}

\author{
Qian Wang, Nick Bennett \\ School of Engineering, University of Portsmouth, UK \\ Email: qian.wang@port.ac.uk
}

Received July 2014

\begin{abstract}
Today, importance of flexibility and reconfigurability needs to be addressed when designing and implementing a cost-effective and responsive manufacturing system. Such a system should be able to accommodate dynamic changes of product varieties and production volumes by maximizing its production capability and minimizing its production costs, this is particularly useful for a SME (small and medium-sized enterprises) to remain competitive in the market. For a manual assembly line, it is always a good practice using a highly skilled workforce that each assembly worker is capable of performing multiple tasks. Ideally, each worker is fully trained to complete assigned tasks of a unit from start to finish. This paper presents a case study of incorporating $5 \mathrm{~S}$ management rules into an assembly system using so-called skillful and dynamic walking workers as a combination of lean management approaches to improve productivity and efficiency of a shop floor production line at a local plant.
\end{abstract}

\section{Keywords}

\section{Lean Management, Lean Production, Assembly Systems}

\section{Introduction}

It has been becoming a popular model for many manufacturing companies to introduce and implement lean approaches into every aspect of manufacturing-related activities. These activities include product design, manufacturing processes and systems planning and production management. One of lean production management techniques is called 5S, which is considered as a lean management method using visual identification and control management rules at a workplace. Major benefits of applying $5 \mathrm{~S}$ techniques to a manufacturing plant were reported as such an improvement of efficiency at a workplace, reduction of wastes, creation of a cleaner and well-organized working environment, and a promotion of employee morale. These benefits can be enhanced by developing a multiple skilled workforce that plays a key role in the success of operating a lean manufacturing system, particularly when such a system involves a great deal of human-centered operations. Ballé et al. [1] suggested that such a manpower production line should also be designed towards a reduction of the seven wastes, i.e., the waste of overproduction, the waste of waiting for parts to arrive, the waste of conveyance, the waste in processing, the waste of inventory, the waste of motion and the waste of rework. This paper presents a case 
study aimed at improving efficiency and productivity of a local manufacturing shop floor by integration of 5S management rules into a manual assembly line using highly skilled walking workers. These workers are capable of performing multiple and/or all the required tasks of a unit by traveling down between stations on the line. Such a system can also be reconfigured easily and quickly as needed to accommodate the fluctuating change of production requirement on a daily basis.

\section{A Lean Management of Using Walking Worker Assembly}

In manufacturing sectors, it is well-known that flexibility and reconfigurability of a manufacturing system has increasingly become important as the system needs to respond quickly to frequent changes of such as product mix and production volume due to a fluctuating demand of the competitive market today. Such a characteristic of flexible and reconfigurable manufacturing systems can also be helpful to maximize systems capability and minimize production costs to compete with other rivals that make similar products. It was reported that approximately one third of all German companies that have invested in highly advanced automaton have recognized that these solutions were not flexible enough and have reduced again their level of automation; 38\% of these companies have reduced automation by taking advantage of a more efficient use of their qualified workforce [2]. These workers can be trained to perform multiple or all the required tasks in a production area leading to a significant improvement in terms of cost, time, quality and capability when dealing with a variety of products over a traditional static allocation of worker(s) to a station in which each worker only performs a single and repetitive task. Thus, capability in manufacturing products with high customer customization is relatively low. Figure 1 illustrates a typical manufacturing system using multifunctional and dynamic walking workers. Within such a system, each worker travels with a partially assembled product downstream and stops at each station carrying out the essential assembly work as scheduled. Each worker is previously trained to be capable of building a product completely from start to end along the line. Under such a 'pull' system, a new item of assembled products enters the line whenever a walking worker is available after a product assembly is completed by this walking worker at the end of the line and this worker then releases the assembled product and moves back to the first station ready to start a new item. Because each item can only travel with one walking worker who works on it by visiting all the stations along the line, the number of items in the system is therefore deterministic and theoretically it cannot be greater than the total number of workers employed on the line. Thus, this type of system inherently prevents unnecessary in-process inventory thereby decreasing the buffer requirement. Moreover, each walking worker on the line cannot be starved because each worker is attached to one item all the time and it is their responsibility for completely assembling a product within an expected cycle time through training, this decreases the loss of labor efficiency and maximizes individual labor utilization in practice. However, the loss of labor efficiency can be made by the idle time, which includes a combination of a possible in-process waiting time on the line and a travel time from each walking worker. Nevertheless, a U-shaped cell as show in Figure $\mathbf{1}$ can minimize the travel time along the line.

\section{Mathematical Analysis}

The following notations are used:

$-m$ : the total number of stations (or machines) on the line.

$-N$ : the total number of walking workers in operating the system.

- PTi: the processing time (fixed) at machine $i$.

- PTi, $j$ : the processing time at machine $i$ for walking worker $j(1 \leq j \leq N)$.

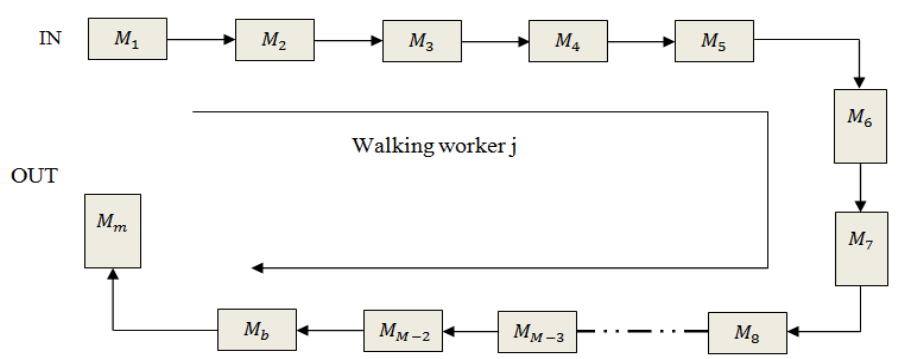

Figure 1. A linear walking worker U-shaped line 
$-I P W T j$ : the in-process waiting time for worker $j(1 \leq j \leq N)$.

$-T T j$ : the average travelling time for walking worker $j(1 \leq j \leq N)$, who travels from the first station to the last station along the line.

-ITj: the total idle time for walking worker $j(1 \leq j \leq N)$ during a completion of a product.

\subsection{Workers with Equal Performance}

In the first case study, we assume that each walking worker has equal efficiency; this is an ideal situation. Shown in Figure 1, we note that machine b (Mb), where b refers to the bottleneck, has the longest processing time. After a period of system warm-up to reach a steady state, from the moment that a walking worker $j$ leaves the bottleneck $\mathrm{Mb}$ to the moment that this worker is about moving into the bottleneck $\mathrm{Mb}$ again (but not in $\mathrm{Mb}$ yet), a total amount of processing (operation) time this walking worker spends in a circuit is given by:

$$
\sum_{\substack{i=1 \\ i \neq b}}^{m}(P T i)=\sum_{i=1}^{m}(P T i)-P T b
$$

Meanwhile, a total amount of time that other walking workers (except walking worker j) spend in terms of processing (operation) times at $\mathrm{Mb}$ is given by:

$$
(N-1) \times P T b
$$

If $(1) \geq(2)$ : there is no in-process waiting time. If $(1)<(2)$ : the in-process waiting time for this walking worker $\mathrm{j}$ is given by: $I P W T j=(2)-(1)=[(N-1) \times P T b]-\left[\sum_{i=1}^{m}(P T i)-P T b\right]$, i.e.,

$$
I P W T j=N \times P T b-\sum_{i=1}^{m} P T i
$$

Thus, the total idle time for this worker $\mathrm{j}$ during the completion of a product is given by:

$$
I T j=I P W T j+2 T T j=N \times P T b-\sum_{i=1}^{m} P T i+2 T T j \text {, i.e., } I T j=N \times P T b-\sum_{i=1}^{m} P T i+2 T T j
$$

Therefore, the amount of time this walking worker needs for producing a unit in a circuit is given below:

$$
I T j+\sum_{i=1}^{m}(P T i) \text { or } N \times P T b+2 T T j
$$

Based on this, the output worker $\mathrm{j}$ produces after a period of run Tp is given by: $T p /[N \times P T b+2 T T j]$

Because we assume that each walking worker has equal efficiency in this case; for a system with $\mathrm{N}$ walking workers, the overall output after a period of run Tp is given by:

$$
N \times\left\{T p /\left[N \times P T b-\sum_{i=1}^{m} P T i+2 T T j+\sum_{i=1}^{m}(P T i)\right]\right\}, \text { i.e., } N \times\{T p /[N \times P T b+2 T T j]\}
$$

\subsection{Workers with Unequal Performance}

In practice, it is impossible that each walking worker has equal efficiency. In this case, the slowest worker may determine the overall output of the line. After a warm-up period, the slowest worker will only possibly encounter the in-process waiting time in front of the bottleneck machine Mb. From the moment that the slowest worker s leaves the bottleneck, this worker needs the following amount of time to arrive to it again in a circuit:

$$
\sum_{\substack{i=1 \\ i \neq b}}^{m}(P T i, s)=\sum_{i=1}^{m}(P T i, s)-P T b, s
$$


Meanwhile, a total amount of time that other walking workers spend at Mb is given by:

$$
\sum_{\substack{j=1 \\ j \neq s}}^{N}(P T b, j)
$$

If $(8) \geq(9)$ : there is no in-process waiting time. If $(8)<(9)$ : the in-process waiting time for the slowest walking worker $\mathrm{s}$ is given by:

$$
\begin{gathered}
I P W T s=(9)-(8)=\left[\sum_{\substack{j=1 \\
j \neq s}}^{N} P T b, j\right]-\left[\sum_{i=1}^{m}(P T i, s)-P T b, s\right], \text { i.e. }, \\
I P W T s=\left[\sum_{j=1}^{N} P T b, j\right]-\left[\sum_{i=1}^{m}(P T i, s)\right]
\end{gathered}
$$

Thus, the total idle time for this slowest worker s during the completion of a product is given by:

$$
\begin{aligned}
& I T s=I P W T s+2 T T s=\left[\sum_{j=1}^{N} P T b, j\right]-\left[\sum_{i=1}^{m}(P T i, s)\right]+2 T T s, i . e ., \\
& I T s=\left[\sum_{j=1}^{N} P T b, j\right]-\left[\sum_{i=1}^{m}(P T i, s)\right]+2 T T s
\end{aligned}
$$

Knowing that a faster worker cannot overtake the slowest worker, therefore, each walking worker will have the same output as the slowest worker can produce. We define $\mathrm{T}$ to be the amount of time each walking worker needs for producing a unit, two cases are possible: If the slowest walking worker does not encounter any in-process waiting time:

$$
T=\sum_{i=1}^{m} P T i, s+2 \mathrm{TTs}
$$

Because IPWTs is 0 , based on equation $10, \mathrm{~T}$ can also be given by:

$$
T=\sum_{j=1}^{N} P T b, j+2 \mathrm{TTs}
$$

If the slowest worker encounters an in-process waiting time before moving into the machine b:

$$
\begin{gathered}
T=I P W T s+\sum_{i=1}^{m} P T i, s+2 \mathrm{TTs} \\
\text { or, } T=\sum_{\substack{j=1 \\
j \neq s}}^{N} P T b, j+2 \mathrm{TTj}
\end{gathered}
$$

Finally, a total amount of in-process waiting time a walking worker $\mathrm{j}$ spends for producing one unit is given by:

$$
I P W T j=T-\sum_{i}^{m} P T i, j-2 \mathrm{TTj}
$$

\section{Implementing 5Ss into Lean Walking Workers Production Lines}

In a manufacturing company, 5Ss are considered as a lean management technique aiming to eliminate manufacturing-related wastes that may obstruct the system performance in terms of line efficiency and productivity, and minimize unnecessary production costs. This is particularly useful for human-centred assembly systems where 
intensive operational tasks are carried out manually and human-errors are often made during an assembly of a unit. The key method of implementing $5 \mathrm{~S}$ management rules into a manual assembly working environment is to make a workplace readable and visible. This can be achieved by arranging visual boards or tags at a workplace with $5 \mathrm{~S}$ concepts embedded into each manufacturing process or manufacturing-related activity. Figure 2 demonstrates a road map as an example for implanting $5 \mathrm{~S}$ at a workstation using walking workers as part of a 5S-enabled shop floor flow line at a local manufacturing plant. Figure 3 illustrates a 3D simulation model of the 5S-enabled shop floor. The simulation model was used for monitoring various scenarios with performance measures based on simulation results before and after the implementation of $5 \mathrm{~S}$ management rules into the production line using walking workers.

\section{Simulation Outputs}

A feasibility study of applying highly skilled walking workers to a shop floor environment was investigated based at a local plant. The company is a small media sized enterprise (SME). In its shop floor, manufacturing processes were performed manually by individual workers who were trained complete a unit by travelling from one workbench station to next workbench station along the line. The simulation result shown in Figure 4 indicates that the original production system has a maximum output of between three and four units per day and the proposed lean production system has a maximum output of five units per day providing a significant increase of productivity. Based on the simulation result shown in Figure 4, it can be seen that the original system requires six walking workers operating on the line simultaneously when the maximum output (three or four units per day) of the line reaches stable, whilst the reconfigured lean system requires four walking workers to achieve the maximum output (i.e., five units per day). This implies that the proposed lean system is more efficient than that of the old system in terms of output per worker per day. For both simulation results shown in Figure 4, it can be seen that the output can be increased or decreased by simply adjusting the number of walking workers on the line, i.e., such a system is very adaptable to the change of a daily or weekly demand of products to be manufactured. Nevertheless, when the line reaches the maximum output, any further increase of the number of workers on the line will not increase but will gradually decrease the overall output. Figure 5 illustrates a trend of the drop of output per worker per day versus the number of additional workers to be put on the line after the line reaches the maximum output.
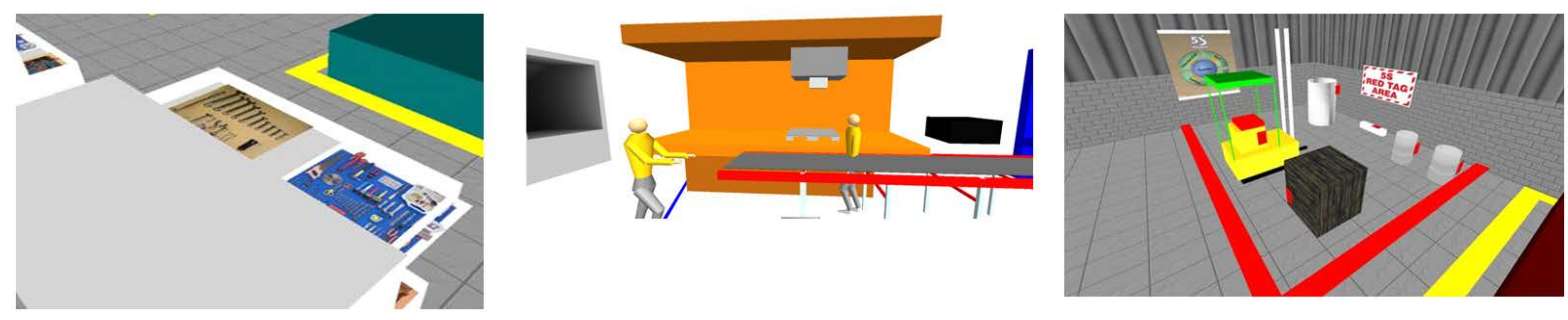

Figure 2. Implementation of 5S management rules into a shop floor environment.

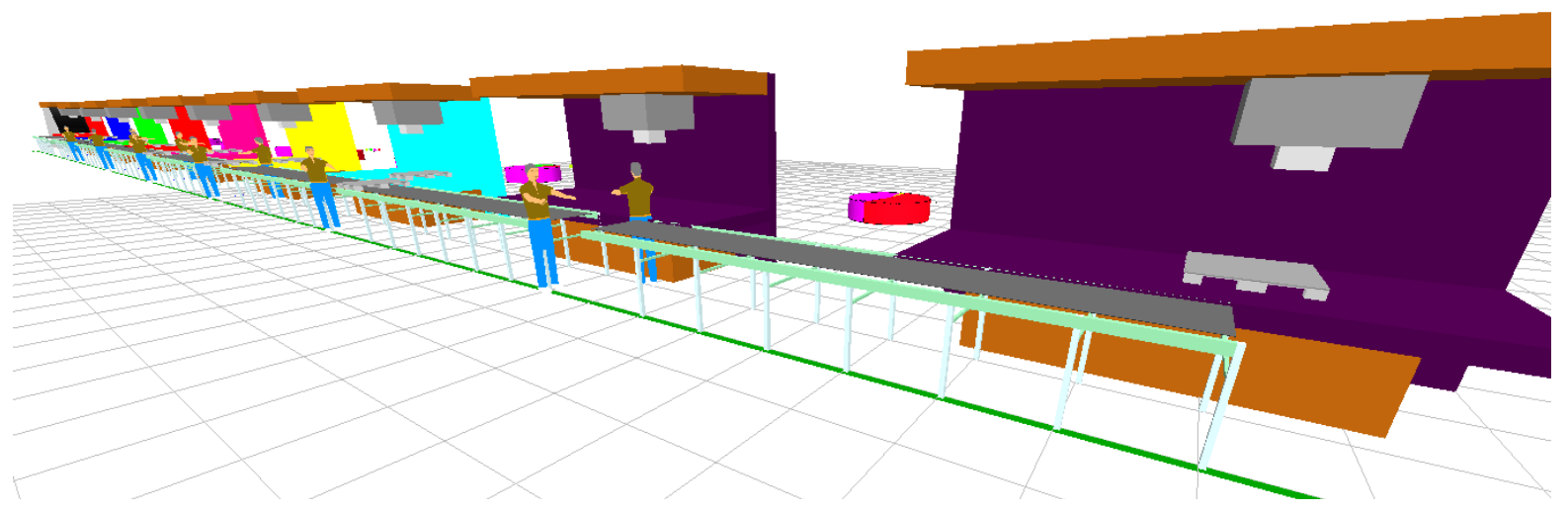

Figure 3. The 5S-enabled walking worker assembly line. 


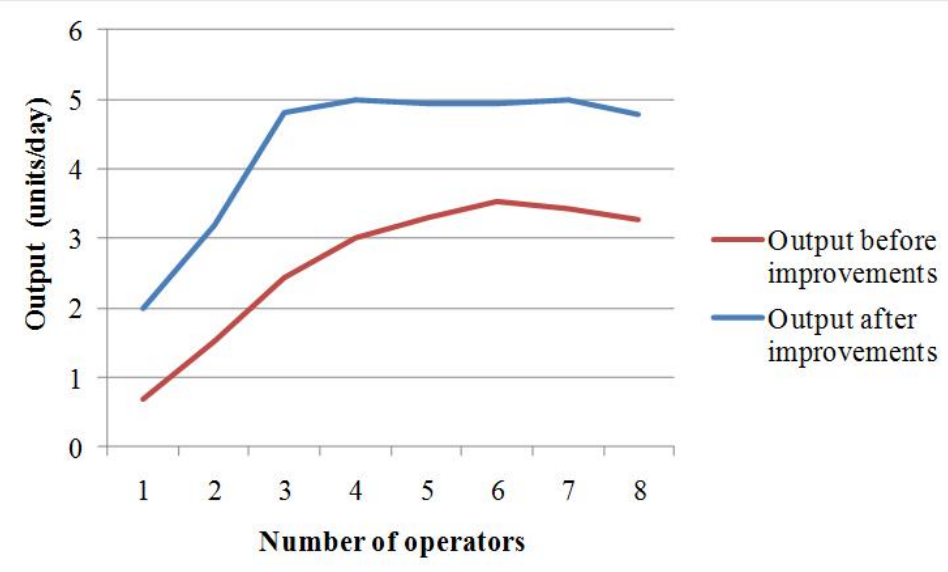

Figure 4. Output vs number of walking workers before and after the implemention of $5 \mathrm{~S}$.

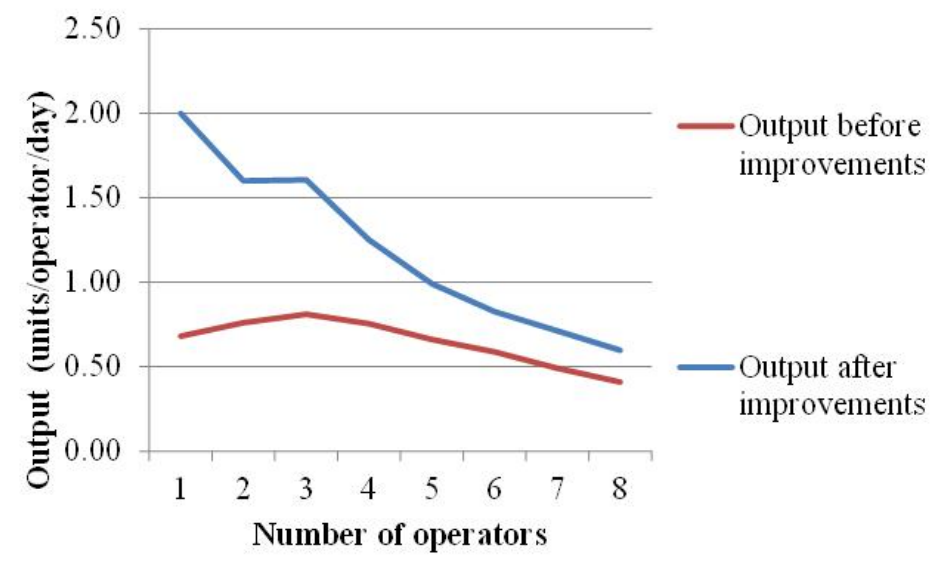

Figure 5. Output per walking worker before and after the implemention of 5S rules.

\section{Summary}

The paper reports a feasibility study of incorporating $5 \mathrm{~S}$ management rules into a production system using wellskilled, flexible and dynamic walking workers at a local manufacturing plant. Both $5 \mathrm{~S}$ and walking workers techniques were applied to the shop floor production as an integrated lean approach aimed at improving efficiency and productivity of the existing system and reducing unnecessary production wastes (costs). The implementation of 5S management rules also improves the visibility and visual control for production as well as safety issues in the shop floor environment. The application of walking workers on the line leads to a significant increase of daily output as well as output per worker per day.

\section{Acknowledgements}

The authors thank Laura Arias, Alexander Bearne and Saleh Alyahya for their contributions to this project; the Director and Production Manager of Wessex Doors for providing valuable information and assistance.

\section{References}

[1] Balle, F. and Balle, M. (2005) Feel the Force of Flexible Manpower. IET Manufacturing Engineer, 84, 20-15.

[2] Bley, H., Reninhart, G., Seliger, G., Bernardi, M. and Korne, T. (2004) Appropriate Human Involvement in Assembly and Disassembly. CIRP Annals, 53, 487-509. http://dx.doi.org/10.1016/S0007-8506(07)60026-2 